\title{
Quantum order by disorder in the Kitaev model on a triangular lattice
}

\author{
George Jackeli ${ }^{1,2, *}$ and Adolfo Avella ${ }^{3,4,5}$ \\ ${ }^{1}$ Institute for Functional Matter and Quantum Technologies, \\ University of Stuttgart, Pfaffenwaldring 57, D-70569 Stuttgart, Germany ${ }^{\dagger}$ \\ ${ }^{2}$ Max Planck Institute for Solid State Research, Heisenbergstrasse 1, D-70569 Stuttgart, Germany \\ ${ }^{3}$ Dipartimento di Fisica "E.R. Caianiello", Università degli Studi di Salerno, I-84084 Fisciano (SA), Italy \\ ${ }^{4}$ CNR-SPIN, UoS di Salerno, I-84084 Fisciano (SA), Italy \\ ${ }^{5}$ Unità CNISM di Salerno, Università degli Studi di Salerno, I-84084 Fisciano (SA), Italy
}

(Dated: November 24, 2015)

\begin{abstract}
We identify and discuss the ground state of a quantum magnet on a triangular lattice with bonddependent Ising-type spin couplings, that is, a triangular analog of the Kitaev honeycomb model. The classical ground-state manifold of the model is spanned by decoupled Ising-type chains, and its accidental degeneracy is due to the frustrated nature of the anisotropic spin couplings. We show how this subextensive degeneracy is lifted by a quantum order-by-disorder mechanism and study the quantum selection of the ground state by treating short-wavelength fluctuations within the linked cluster expansion and by using the complementary spin-wave theory. We find that quantum fluctuations couple next-nearest-neighbor chains through an emergent four-spin interaction, while nearest-neighbor chains remain decoupled. The remaining discrete degeneracy of the ground state is shown to be protected by a hidden symmetry of the model.
\end{abstract}

PACS numbers: 75.10.Jm, 75.30.Ds, 75.30.Et

Frustrated magnets, systems in which every pairwise exchange interaction cannot be simultaneously satisfied, are characterized by accidental degeneracies between various order patterns ${ }^{1}$. Often, these accidental degeneracies are lifted via an order-by-disorder mechanism, driven by thermal and/or quantum fluctuations, selecting an unique ground state $^{2-4}$. In highly frustrated quantum magnets, those with extensive degeneracy, e.g., the isotropic spin one-half kagomé and pyrochlore antiferromagnets (AF), the order-by-disorder mechanism is inactive and they remain disordered down to the lowest temperatures, realizing so-called quantum spin liquids (QSL) in their ground states ${ }^{1}$.

In Mott insulators, with unquenched orbital moments and strong spin-orbit coupling, bond-dependent Isingtype interactions may dominate over the conventional Heisenberg term ${ }^{5-7}$. In turn, such Ising-type couplings, even being ferromagnetic (FM), can frustrate a longrange magnetic order and stabilize a QSL state ${ }^{8}$. The most celebrated model realizing the above scenario is the exactly solvable Kitaev honeycomb model ${ }^{9}$. In this model, nearest-neighbor (NN) spins are coupled by Isingtype terms and the three non-equivalent bonds of the honeycomb lattice host different components of the spin one-half operators. Its ground state is a QSL with fractionalized fermionic excitations ${ }^{9}$.

Following a theoretical proposal ${ }^{7}$ for a possible realization of the Kitaev honeycomb model in iridates $A_{2} \mathrm{IrO}_{3}(A=\mathrm{Na}, \mathrm{Li})$, various extensions of the model have been studied in connection to experiments ${ }^{10-16}$ on actual materials. These model extensions include terms like: the isotropic Heisenberg exchange (the so-called Kitaev-Heisenberg (KH) model ${ }^{17-21}$, furtherneighbor couplings ${ }^{21-23}$, and additional symmetryallowed anisotropies ${ }^{24-28}$. The resulting theoretical phase diagrams are characterized by various ordered phases (including those seen experimentally) and by a finite stability window for QSL around the Kitaev limit.

Recently, a triangular analog of the $\mathrm{KH}$ model ${ }^{29}$ for classical $^{30}$ and quantum ${ }^{31,32}$ spins has been studied numerically. The obtained rich phase diagram includes a $\mathbb{Z}_{2}$-vortex crystal phase near the AF Heisenberg limit, and a nematic phase of decoupled Ising chains with subextensive degeneracy at the Kitaev limit ${ }^{30,31}$. In addition, a chiral spin-liquid phase has been proposed close to the antiferromagnetic Kitaev limit ${ }^{32}$.

Here, we study analytically the Kitaev model on the triangular lattice and solve the puzzle of its ground state by analyzing the effects of quantum fluctuations within both the linked-cluster expansion, ${ }^{33}$ combined with degenerate perturbation theory, and the linear spin-wave theory. We show that such a deceptively simple model, once realized on a triangular lattice, becomes the host of very interesting and unexpected order-by-disorder effects such as: the quantum selection of the easy axes, the emergence of a specific four-spin interaction, the reduction of the sub-extensive degeneracy of the nematic ground state manifold down to a discrete one protected by a hidden symmetry of the model.

\section{THE MODEL}

We consider a triangular lattice lying in the $(1,1,1)$ plane of the spin-quantization frame [see Fig. 1(a)] and label by $(\gamma)(=x, y, z)$ its three non-equivalent $\mathrm{NN}$ bonds spanned by the lattice vectors $\mathbf{a}_{x}=(1 / 2,-\sqrt{3} / 2), \mathbf{a}_{y}=$ $(1 / 2, \sqrt{3} / 2)$ and $\mathbf{a}_{z}=(1,0)$, respectively. On a $(\gamma)$-bond, the one perpendicular to the $\gamma$ spin-quantization axis, only the $S_{\mathbf{i}}^{\gamma}$ components of the spin one-half operators 
(a)

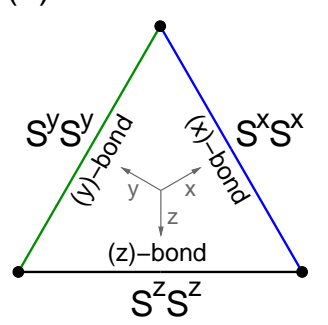

(b)

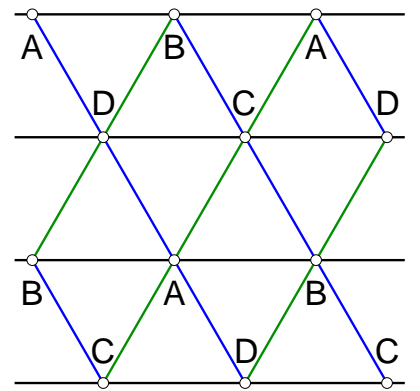

(c)

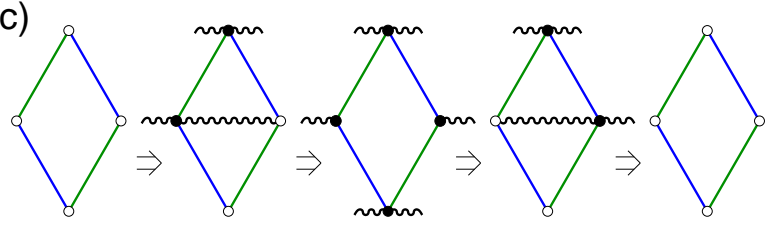

Figure 1. (color online). (a) Ising-type spin couplings on the three non-equivalent bonds of the triangular lattice of model (1). The lattice lies in the $(1,1,1)$ plane of the spinquantization axes. On a $(\gamma)$-bond, the one perpendicular to the $\gamma(=x, y, z)$ spin quantization axis, only the $\gamma$-components of neighboring spins are coupled. (b) Four-sublattice structure of the triangular lattice used for the unitary transformations discussed in the text. (c) Sketch of the $4^{\text {th }}$ order perturbation process leading to the coupling, via quantum fluctuations, of the four spins siting around a diamond [see Eq. (3)]. In the virtual states, the location of the misaligned spins (spin-flips are performed in pairs at one of the 4 bonds of the diamond at each step: top-left $\Rightarrow$ bottom-right $\Rightarrow$ bottomleft $\Rightarrow$ top-right) are shown by filled circles and the wavy lines mark the broken $(z)$-bonds.

$\mathbf{S}_{\mathbf{i}}$ are coupled by a Ising-type interaction [see Fig. 1(a)], and the corresponding Hamiltonian takes the following form

$$
\mathcal{H}=-\sum_{\mathbf{i}, \gamma} K_{\gamma} S_{\mathbf{i}}^{\gamma} S_{\mathbf{i}+\mathbf{a}_{\gamma}}^{\gamma}
$$

In model (1), the signs of the $K_{\gamma}$ couplings can be individually flipped by means of a canonical transformation. For instance, to flip the sign of $K_{z}$ independently from the signs of the other two couplings, $K_{x}$ and $K_{y}$, one needs to perform spin rotations around the $y$ axis by an angle $180^{\circ}$ on sites belonging to the sublattices B and C [see Fig. 1(b)], i.e., $\left[\left(S_{\mathbf{i}}^{x}, S_{\mathbf{i}}^{y}, S_{\mathbf{i}}^{z}\right) \rightarrow\left(-S_{\mathbf{i}}^{x}, S_{\mathbf{i}}^{y},-S_{\mathbf{i}}^{z}\right)\right.$ for $\mathbf{i} \in \mathrm{B} \oplus \mathrm{C}]$. The signs of $K_{x}\left(K_{y}\right)$ can be flipped independently in the very same way by performing $180^{\circ}$ spin rotations around $z(x)$ axis on the sublattices B and D (A and B). In what follows, without any loss of generality, we consider all $K_{\gamma}$ to be positive (FM couplings).

\section{GROUND STATE MANIFOLD}

In the isotropic FM case $K_{\gamma}=K>0$, the classical ground-state energy is simply proportional to $\mathbf{M}^{2}$ where $\mathbf{M}=\left\langle\mathbf{S}_{\mathbf{i}}\right\rangle$. This accidental symmetry implies that the ordered moment $\mathbf{M}$ can be freely rotated, i.e., no preferred axis exists. Moreover, the coupling between NN chains, along any of the three lattice directions (e.g., spanning along $(z)$-bonds), does not involve the corresponding projections of the spins (e.g., $S_{\mathbf{i}}^{z}$ ). Therefore, these latter projections of the spins can be freely flipped along any of those chains individually ${ }^{30,31}$. This leads to an additional $2^{L}$-fold degeneracy, where $L$ is the linear size of the system. In the anisotropic case, when the couplings $K_{\gamma}$ are different from one another, the easy axis is dictated by the strongest coupling (e.g., $z$ axis for $\left.\left|K_{z}\right|>\left|K_{x}\right|,\left|K_{y}\right|\right)$. However, the ground-state manifold still has a sub-extensive degeneracy as it is characterized by completely decoupled either FM (for $K_{z}>0$ ) or $\mathrm{AF}$ (for $K_{z}<0$ ) chains along $(z$ )-bonds. Such a subextensive degeneracy is inherent to models with Ising- or compass-type bond-dependent anisotropies ${ }^{8}$.

In principle, these accidental classical degeneracies, not being related to apparent symmetries, can be lifted by quantum fluctuations. We would need to calculate the energy corrections due to zero-point quantum fluctuations (e.g., within the spin-wave theory) for each degenerate classical ground state and single out a ground state for which the corrected energy is minimized. For an infinitely degenerate manifold this is obviously not feasible and we need to resort to some other procedure. The linked-cluster expansion, ${ }^{33}$ combined with degenerate perturbation theory, allows to compute quantum corrections to a ground-state energy from short-wavelength quantum fluctuations and to identify the mechanism for quantum selection of the ground state. ${ }^{34-39}$

\section{QUANTUM SELECTION OF THE GROUND STATE}

\section{A. Easy axes}

In the isotropic case $K_{\gamma}=1$, we consider a FM state with the ordered moment $\mathbf{M}$ pointing in a generic direction identified by the unit vector $\mathbf{m}=\left(m_{x}, m_{y}, m_{z}\right)=$ $(\sin \theta \cos \phi, \sin \theta \sin \phi, \cos \theta)$. Then, we rotate the spinquantization frame $x y z$ of the Hamiltonian (1) to a new frame $x^{\prime} y^{\prime} z^{\prime}$ in which $\mathbf{m} \| z^{\prime}$. The transformed Hamiltonian on $\mathrm{NN} i j$ bond includes various terms in the new spin-quantization frame: the $S_{i}^{z^{\prime}} S_{j}^{z^{\prime}}$ terms represent the unperturbed (mean-field) Hamiltonian and the remaining ones, those creating misaligned spins at the cost of a mean-field energy, are treated as perturbations. At the second order in the perturbation expansion, the terms creating only one spin-flip, e.g., $S_{i}^{x^{\prime}} S_{j}^{z^{\prime}}$, give energy corrections that sum up to zero. Only the terms inducing two spin-flips on a given $(\gamma)$-bond give a cumulative finite energy correction depending on the direction of $\mathbf{m}$. The creation/annihilation amplitude for two misaligned spins on a $(\gamma)$-bond is $T_{\gamma}=\left(1-m_{\gamma}^{2}\right) / 4$ with a corresponding energy cost $\Delta_{\gamma}=\left(2-m_{\gamma}^{2}\right)$. This gives the following quantum energy correction per site: 


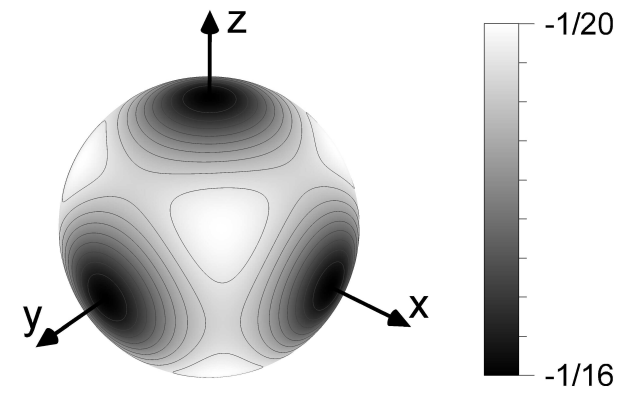

Figure 2. Color map of the second-order quantum energy correction (2) for an arbitrary direction $\mathbf{m}$ of the ordered moment. The minimum energy is achieved for $\mathbf{m}$ pointing along one of the spin-quantization axes.

$$
\delta E^{(2)}(\mathbf{m})=-\sum_{\gamma} \frac{T_{\gamma}^{2}}{\Delta_{\gamma}} \simeq-\frac{3}{64}\left(1+\frac{1}{6} \sum_{\gamma} m_{\gamma}^{4}\right)
$$

In Fig. 2, we report the second-order quantum energy correction (2) for an arbitrary direction $\mathbf{m}$ of the ordered moment via a color map. Each point on the sphere stands for a specific direction of $\mathbf{m}$ within the original spin-quantization frame $x y z$ and the color scale gives the corresponding value of (2). It is evident that the minimum of the energy is realized when $\mathbf{m}$ points along one of the spin-quantization axes implying that they are selected by quantum corrections as the easy axes. This can be also seen from the result of a naive expansion, the last term in Eq. (2), explicitly showing how a fourth-order cubic anisotropy emerges from quantum fluctuations.

The above result explains and quantifies the order-bydisorder selection of the easy axes numerically found in Refs. 30 and 31 by means of classical Monte Carlo and of density matrix renormalization group (DMRG) methods, respectively, and also agrees with previous studies performed on models similar to (1), but realized on other lattices ${ }^{17,40-42}$. This study is complementary to the spinwave analysis performed in Ref. 31 around the Heisenberg limit of the FM KH model on a triangular lattice. In that case, a finite Kitaev coupling leads to the selection of easy axes and breaks the accidental $O(3)$ symmetry down to $\mathbb{Z}_{6}$. In the present case, the degenracy of the ground state remains sub-extensive at $3 \times 2^{L}$.

\section{B. Coupling between chains}

Both in the isotropic case $K_{\gamma}=K$ (choosing $z$ as easy axis) and in the anisotropic case $\left|K_{z}\right| \geq\left|K_{x}\right|,\left|K_{y}\right|$, the ground-state manifold is spanned by decoupled either FM (for $K_{z}>0$ ) or $\mathrm{AF}$ (for $K_{z}<0$ ) chains along $(z)$-bonds. We now compute, within the linked-cluster expansion $^{33}$, the quantum corrections induced by the $K_{x}$ and $K_{y}$ terms, and find the related effective couplings between chains. The expansion parameter scales as $K_{x(y)} / z K_{z}(\mathrm{z}=3$ being the number of $\mathrm{NNs})$, and the results we derive are valid in the isotropic case $K_{\gamma}=K$ too. The $K_{x}$ and $K_{y}$ terms generate fluctuations out of the classical ground state by creating/annihilating pairs of misaligned spins on the corresponding bonds. Accordingly, the linked graphs relevant to the perturbation expansion are those composed by $(x)$ - and/or $(y)$ bonds. Open linked graphs composed of $n$ bonds contribute to the $(2 n)^{t h}$ leading order, while closed linked graphs composed of $n$ bonds contribute to the $n^{\text {th }}$ leading order. Given the obvious absence of $(z)$-bonds in the perturbation expansion, there is no loop-like cluster composed of an odd number of bonds and hence no perturbative odd-order correction exist. In the $2^{\text {nd }}$ order, there is no coupling between chains but a reduction of the ground state energy by $-\frac{1}{32} \frac{K_{x}^{2}+K_{y}^{2}}{\left|K_{z}\right|}$. In the $4^{\text {th }}$ order, straight 3 -site clusters, composed of two $(x)$ - or two $(y)$ - bonds, give just a reduction of the ground state energy by $-\frac{1}{2048} \frac{\left(K_{x}^{2}+K_{y}^{2}\right)^{2}}{\left|K_{z}^{3}\right|}$ and again do not couple chains. The other two types of 3 -site clusters, composed of $(x)$ and $(y)$ - bonds, with $\pi / 3$ or $2 \pi / 3$ angles in between, give no contribution at all because the $\mathcal{H}^{(x)}$ and $\mathcal{H}^{(y)}$ bond Hamiltonians with a common vertex anticommute and they always come in permuted pairs. The $4^{\text {th }}$ order correction coming from a diamond-shape 4-site cluster [see Fig. 1(c)] give instead a coupling between pairs of spins belonging to next-nearest-neighbor (NNN) chains in the following form

$$
\delta \mathcal{H}^{(4)}=-\frac{1}{24} \frac{K_{x}^{2} K_{y}^{2}}{\left|K_{z}^{3}\right|} \sum_{\mathbf{i}}\left(S_{\mathbf{i}}^{z} S_{\mathbf{i}+\mathbf{a}_{z}}^{z}\right) S_{\mathbf{i}+\mathbf{a}_{x}}^{z} S_{\mathbf{i}+\mathbf{a}_{y}}^{z}
$$

where the sites $\mathbf{i}+\mathbf{a}_{x}$ and $\mathbf{i}+\mathbf{a}_{\mathbf{y}}$ belong to NNN chains, and they are the ends of the longer diagonal of the $d i$ amond cluster [see Fig. 1(c)]. It is worth noting that $S_{\mathbf{i}}^{z} S_{\mathbf{i}+\mathbf{a}_{z}}^{z}$ is just $\frac{1}{4}$ for $K_{z}>0$ and $-\frac{1}{4}$ for $K_{z}<0$ leading to a coupling between NNN chains of the same sign of the one acting along the chains. This does not fully lift the degeneracy as, at this order, the two sublattices formed by NN chains remain decoupled. The degeneracy is fourfold ( 3 times four-fold for $K_{x}=K_{y}=K_{z}$ ). Actually, we found that this degeneracy is dictated by a hidden symmetry of the model. This hidden symmetry is uncovered by a four-sublattice unitary transformation from Ref. 5. This transformation leaves the Hamiltonian (1) unchanged, but flips the sign of the $z$ components of the spins on only one of the two sublattices. We divide the triangular lattice in 4 sublattices, as shown in Fig. 1(b), and perform the following local spin-rotations: by $180^{\circ}$ on sublattices $\mathrm{B}, \mathrm{C}$, and $\mathrm{D}$ around $z, y$, and $x$ axes, respectively, while keeping the spins on the $A$ sublattice in the original frame. This transformation leaves the Hamiltonian (1) unchanged. On the other hand, the net effect on a state with spins ordered along the $z$ direction is to flip the sign of the $z$ component of every second chain, the chains belonging to the $\mathrm{C} \oplus \mathrm{D}$ sublattice, showing 
that NN chains are completely decoupled as any relative order between them leads to the same energy.

\section{Comparison to numerics}

Very recently, Becker et. $a l,{ }^{31}$ used the DMRG method to compute the ground state and the first excited states of the Kitaev Hamiltonian (1) in the AF isotropic case $\left(K_{\gamma}=K<0\right)$ on finite clusters with open boundary conditions. The considered clusters are strips of 3 and 4 chains of length $L \leq 14$, cut out from a triangular lattice (see Figs. 12 and 13 in Ref. 31). The spatial anisotropy of such clusters breaks the original symmetry of the triangular lattice and forces the spins to order $\mathrm{AF}$ along the longer direction (e.g., along the (z)-bonds) and, correspondingly, in the like spin component $\left(S^{z}\right)$. Measuring the spin correlation functions across the system, they found AF correlations between the NNN chains and no correlations between NN ones, in agreement with our analysis. Moreover, for the largest analyzed system, the numerically found gap to the first excited state, featuring FM correlations between NNN chains, amounts to $0.0055|K|$ per diamond, which is again in very good agreement with the value $1 / 192|K| \simeq 0.0052|K|$ predicted by Eq. (3).

\section{SPIN-WAVE THEORY}

We now apply the linear spin-wave theory to the Hamiltonian (1). In order to compare the results obtained by the linear spin-wave theory with those obtained by the linked-cluster expansion, we will focus on the case $K_{z} \geq K_{x}, K_{y}>0$ and consider two states that are degenerate in the classical limit. These are the FM and stripy AF states [shown as right panels in Fig. 3] with spin ordering along the $z$ axis. Within spin-wave theory we find one branch in the FM state

$$
\omega^{2}(\mathbf{q})=\left(K_{z}-K_{x} c_{x}\right)\left(K_{z}-K_{y} c_{y}\right),
$$

and four branches in the stripy AF state

$$
\omega_{1,2}^{2}(\mathbf{q})=K_{z}^{2} \pm \sqrt{K_{z}^{2}\left(K_{x}^{2} c_{x}^{2}+K_{y}^{2} s_{y}^{2}\right)-K_{x}^{2} K_{y}^{2} c_{x}^{2} s_{y}^{2}}
$$

where $c_{x}=\cos \mathbf{q} \cdot \mathbf{a}_{x}, c_{y}=\cos \mathbf{q} \cdot \mathbf{a}_{y}, s_{x}=\sin \mathbf{q} \cdot \mathbf{a}_{x}$ and $s_{y}=\sin \mathbf{q} \cdot \mathbf{a}_{y}$. The other two branches, $\omega_{3,4}(\mathbf{q})$, are obtained from $\omega_{1,2}(\mathbf{q})$ by the exchanges $c_{x} \leftrightarrow c_{y}$ and $s_{x} \leftrightarrow s_{y}$.

Fig. 3 shows the color map of the obtained spin-wave excitation spectra for $K_{\gamma}=1$ in both the FM state and the stripy AF state. In this latter case, only one of the two degenerate lower branches is reported. The corresponding magnetic structures are sketched on the right. In both cases, the excitation spectra are well defined over the entire Brillouin zone, confirming that the FM and
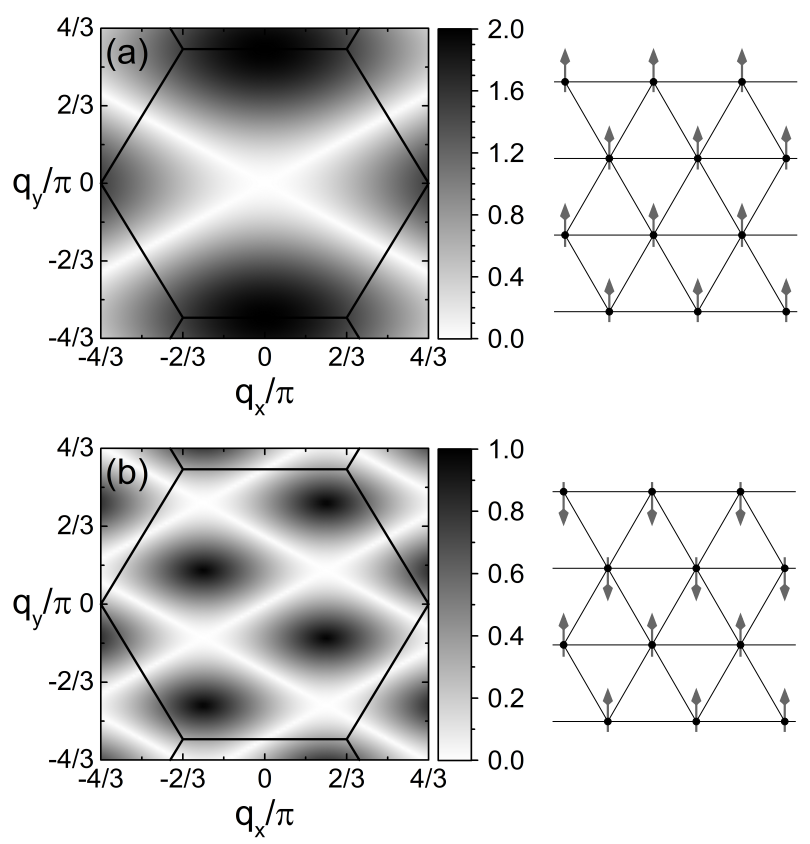

Figure 3. Color map of the spin-wave excitation spectrum of model (1) in (a) the FM state Eq. (4) and (b) the stripy AF state Eq. (5), for $K_{\gamma}=1$. Only one of the lower branches is shown in (b). Hexagons mark the crystallographic Brillouin zone. The right panels report the corresponding magnetic structures.

the stripy AF states do indeed minimise the classical energy. Moreover, the lines of nodes, related to the subextensive degeneracy of the classical manifold discussed above, are clearly visible. Comparing the zero-point spinwave energies obtained from Eqs. (4) and (5), we find that the FM state is always favored against the stripy AF state, in agreement with the linked-cluster expansion result. Moreover, by expanding the spin-wave excitation spectra in Eqs. 4 and 5 in terms of small $K_{x, y} / K_{z}$, we find analytically the difference between the zero-point energies of the FM and the stripy AFM states to be $\delta E_{\mathrm{sw}}^{(4)}=-\frac{3}{4} \times \frac{1}{192} \frac{K_{x}^{2} K_{y}^{2}}{K_{z}^{3}}$, which is in agreement with the prediction of Eq. (3) except for a multiplicative factor $\frac{3}{4}$, whose presence can be anyway readily explained. The linear spin-wave theory does not take into account the interactions between misaligned spins. Therefore, in the virtual state shown as the middle diamond in Fig. 1(c), 8 broken $(z)$-bonds are counted in the linear spin-wave theory instead of the actual 6 broken $(z)$-bonds shown in Fig. 1(c).

In conclusion, we have discussed quantum order-bydisorder in the Kitaev model on the triangular lattice within the linked-cluster expansion and the complementary spin-wave theory, and clarified the true nature of the ground state of this frustrated quantum spin model. In particular, we have shown (i) the presence of a mechanism of quantum selection of easy axes, (ii) the emergence of a four-spin interaction that reduces the sub-extensive 
degeneracy of the ground state manifold $\left(3 \times 2^{L}\right)$ down to a discrete one $\left(3 \times 2^{2}\right)$, and (iii) the existence of a hidden symmetry of the model that protects this latter degeneracy. The present analytical study explains and quantifies the results of numerical simulations ${ }^{31}$. The developed scheme, that makes explicit links between degenerate perturbation theory and spin-wave analysis, can be applied to other quantum spin models in which spin frustration is driven by anisotropic spin couplings.

We thank A.F. Bangura, M. Becker, M. Daghofer, M. Hermanns, G. Khaliullin, S. Trebst and E.A. Yelland for discussions and comments. AA acknowledges kind hospitality at the Max Planck Institute for Solid State Research, Stuttgart, Germany.
* Also at E. Andronikashvili Institute of Physics, 0177 Tbilisi, Georgia

† G.Jackeli@fkf.mpg.de

¥ avella@physics.unisa.it

1 L. Balents, Nature 464, 199 (2010).

2 J. Villain, R. Bidaux, J.-P. Carton, and R. Conte, J. Phys. France 41, 1263 (1980).

3 E. F. Shender, Zh. Eksp. Teor. Fiz. 83, 326 (1982), Sov. Phys. JETP 56, 178 (1982).

4 For the discussions on experimental manifestation of quantum order-by-disorder see M. E. Zhitomirsky, M. V. Gvozdikova, P. C. W. Holdsworth, and R. Moessner, Phys. Rev. Lett. 109, 077204 (2012); L. Savary, K. A. Ross, B. D. Gaulin, J. P. C. Ruff, and L. Balents, ibid. 109, 167201 (2012).

5 G. Khaliullin, Prog. Theor. Phys. Suppl. 160, 155 (2005).

${ }^{6}$ G. Chen and L. Balents, Phys. Rev. B 78, 094403 (2008).

7 G. Jackeli and G. Khaliullin, Phys. Rev. Lett. 102, 017205 (2009).

${ }^{8}$ For a review see Z. Nussinov and J. van den Brink, "Compass and Kitaev models - Theory and Physical Motivations," (2013), arXiv:1303.5922 [cond-mat.str-el]; Z. Nussinov and J. van den Brink, Rev. Mod. Phys. 87, 1 (2015).

9 A. Kitaev, Annals of Physics 321, 2 (2006).

10 Y. Singh and P. Gegenwart, Phys. Rev. B 82, 064412 (2010).

11 X. Liu, T. Berlijn, W.-G. Yin, W. Ku, A. Tsvelik, Y.-J. Kim, H. Gretarsson, Y. Singh, P. Gegenwart, and J. P. Hill, Phys. Rev. B 83, 220403 (2011).

12 S. K. Choi, R. Coldea, A. N. Kolmogorov, T. Lancaster, I. I. Mazin, S. J. Blundell, P. G. Radaelli, Y. Singh, P. Gegenwart, K. R. Choi, S.-W. Cheong, P. J. Baker, C. Stock, and J. Taylor, Phys. Rev. Lett. 108, 127204 (2012).

13 Y. Singh, S. Manni, J. Reuther, T. Berlijn, R. Thomale, W. Ku, S. Trebst, and P. Gegenwart, Phys. Rev. Lett. 108, 127203 (2012).

14 F. Ye, S. Chi, H. Cao, B. C. Chakoumakos, J. A. Fernandez-Baca, R. Custelcean, T. F. Qi, O. B. Korneta, and G. Cao, Phys. Rev. B 85, 180403 (2012).

15 H. Gretarsson, J. P. Clancy, Y. Singh, P. Gegenwart, J. P. Hill, J. Kim, M. H. Upton, A. H. Said, D. Casa, T. Gog, and Y.-J. Kim, Phys. Rev. B 87, 220407 (2013).

16 S. H. Chun, J.-W. Kim, J. Kim, H. Zheng, C. C. Stoumpos, C. D. Malliakas, J. F. Mitchell, K. Mehlawat, Y. Singh, Y. Choi, T. Gog, A. Al-Zein, M. Moretti Sala, M. Krisch, J. Chaloupka, G. Jackeli, G. Khaliullin, and B. J. Kim, Nat. Phys. 11, 462 (2015).

17 J. Chaloupka, G. Jackeli, and G. Khaliullin, Phys. Rev. Lett. 105, 027204 (2010).
18 H.-C. Jiang, Z.-C. Gu, X.-L. Qi, and S. Trebst, Phys. Rev. B 83, 245104 (2011).

19 J. Reuther, R. Thomale, and S. Trebst, Phys. Rev. B 84, 100406 (2011).

20 C. C. Price and N. B. Perkins, Phys. Rev. Lett. 109, 187201 (2012).

21 J. Chaloupka, G. Jackeli, and G. Khaliullin, Phys. Rev. Lett. 110, 097204 (2013).

22 I. Kimchi and Y.-Z. You, Phys. Rev. B 84, 180407 (2011).

23 J. Reuther, R. Thomale, and S. Rachel, Phys. Rev. B 90, 100405 (2014).

${ }^{24}$ V. M. Katukuri, S. Nishimoto, V. Yushankhai, A. Stoyanova, H. Kandpal, S. Choi, R. Coldea, I. Rousochatzakis, L. Hozoi, and J. van den Brink, New Journal of Physics 16, 013056 (2014).

25 J. G. Rau, E. K.-H. Lee, and H.-Y. Kee, Phys. Rev. Lett. 112, 077204 (2014).

${ }^{26}$ Y. Yamaji, Y. Nomura, M. Kurita, R. Arita, and M. Imada, Phys. Rev. Lett. 113, 107201 (2014).

27 Y. Sizyuk, C. Price, P. Wölfle, and N. B. Perkins, Phys. Rev. B 90, 155126 (2014).

28 J. c. v. Chaloupka and G. Khaliullin, Phys. Rev. B 92, 024413 (2015).

29 The KH triangular model is an extention of the anisotropic spin model originally proposed and studied in Ref. 5 in the context of sodium cobaltates.

30 I. Rousochatzakis, U. K. Rössler, J. van den Brink, and M. Daghofer, "Z_2-vortex lattice in the ground state of the triangular Kitaev-Heisenberg model," (2012), arXiv:1209.5895 [cond-mat.str-el].

31 M. Becker, M. Hermanns, B. Bauer, M. Garst, and S. Trebst, Phys. Rev. B 91, 155135 (2015).

32 K. Li, S.-L. Yu, and J.-X. Li, New J. Phys. 17, 043032 (2015).

33 M. P. Gelfand and R. R. P. Singh, Adv. Phys. 49, 93 (2000).

34 M. W. Long, Journal of Physics: Condensed Matter 1, 2857 (1989).

35 M. T. Heinilä and A. S. Oja, Phys. Rev. B 48, 7227 (1993).

36 D. L. Bergman, R. Shindou, G. A. Fiete, and L. Balents, Phys. Rev. B 75, 094403 (2007).

37 V. S. Maryasin and M. E. Zhitomirsky, Phys. Rev. B 90, 094412 (2014).

38 A. L. Chernyshev and M. E. Zhitomirsky, Phys. Rev. Lett. 113, 237202 (2014).

39 M. E. Zhitomirsky, J. Phys.: Conf. Ser. 592, 012110 (2015).

40 G. Khaliullin, Phys. Rev. B 64, 212405 (2001).

41 E. Sela, H.-C. Jiang, M. H. Gerlach, and S. Trebst, Phys. Rev. B 90, 035113 (2014).

42 Y. Sizyuk, N. B. Perkins, and P. Wölfle, Phys. Rev. B 92, 155131 (2015). 